\title{
The dynamic model of agricultural land structure on the space images in the precision agriculture tasks
}

\author{
Ruslan V. Brezhnev ${ }^{1 *}$ and Yuriy A. Maglinets ${ }^{1}$ \\ ${ }^{1}$ The Institute of Space and Information Technology of Siberian Federal University 660074, Russian \\ Federation, Krasnoyarsk, Akademika Kirenskogo Str. 26/1
}

\begin{abstract}
The study is devoted to the analysis of specific features of spacial objects referred to "agricultural land" class in Central Siberia according to the results of the Earth remote sensing from the space for information support in the precision agriculture tasks. The subject of the study is temporal variability of spectral, textural and geometrical features of a land area with homogenous vegetation (hereinafter agricultural contour). During the vegetation period the agricultural contour is subject to changes caused by a combination of natural and antropogenic factors. These factors are the result of the natural course of vegetation (change of phenological phases), weather conditions and agricultural engineering measures implemented. They typically cause the change of the spacial structure of the agricultural contour resulting in non-homogenous vegetation of an agricultural crop within the agricultural contour.
\end{abstract}

\section{Introduction}

Aerospace monitoring methods have become widely used in agricultural activity. It applies to the whole series of thematic tasks: estimation of crop rotation field state and utilization, parameters of soil fertility and degradation, state of vegetation cover on plough lands, deposits, hay fields and grazing lands, crop yield forecasting, etc.

One of the most perspective areas in which the results of space monitoring can be used in agriculture is precision agriculture, according to which an agricultural land is treated as a special object with non-homogeneous dynamically changing structure [1,2].

Localization of inhomogeneities and determination of a state thereof, together with the data of field studies and information on chemical composition of soils, is important for the support of farmers when making a decision on spotted application of specialized technical means for differentiated treatment of the required non-homogeneous land areas depending on their state.

\footnotetext{
${ }^{*}$ Corresponding author: brezhnev.ruslan@gmail.com
} 


\section{The dynamic model of agricultural land structure}

A state of an F-object means a result of influence exerted by numerous regional conditions of natural and climatic and antropogenic character determining the development of an agricultural crop $k^{i}$, possessing its own temporal development model Tm. On a digital space image, a state of an object means values of spectral features calculated within the defined time intervals and their reference values and context-dependent descriptions that enable to correlate a localized land area $w_{i}$ of the image area $\Theta_{i}$ with the state $f^{i} \in F$.

From the point of view of precision agriculture, a state of a facility cannot be considered exclusive of its local states caused by the deviation of a reference development pathway of the object defined by a time sequence of change of phenological phases $-\varphi=\left\{\varphi^{i}\right\}$ and agro-engineering measures (AEM) $-E=\left\{e^{i}\right\}$. Phenological phases define a set of states $f^{v i}$ and AEM - $f^{w i}$. Thus, every state $f^{i} \in F$ is a composite one and can be presented in the form of a state and transition diagram $G_{i}$ :

$$
G_{i}=(F, R)
$$

where $F$ is a set of states of the object:

$$
F=f^{v i} \cup f^{w i}
$$

$R$ is a set of state transitions:

$$
R=r^{1} \cup r^{2} \cup r^{3}
$$

$r^{1}$ is a set of operators describing a natural change of phenological phases of agricultural crop development in time; $r^{2}$ is a set of operators describing abnormal features in the development of an agricultural crop; and $r^{3}$ is a set of operators modelling the implementation of AEM.

In the process of space monitoring organization, NDVI spectral index is used to qualitatively characterize a change in the vegetation activity and to establish bonds between index values fixed in the image and elements of the set F. Figure 1 gives an example of an agricultural land object with non-homogeneous structure shot at different time periods.

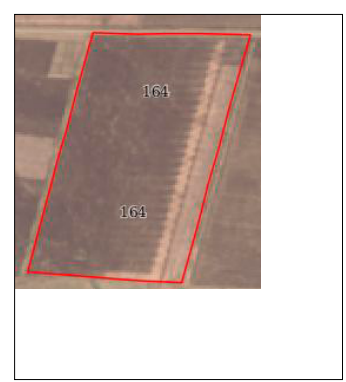

21.05 .2018

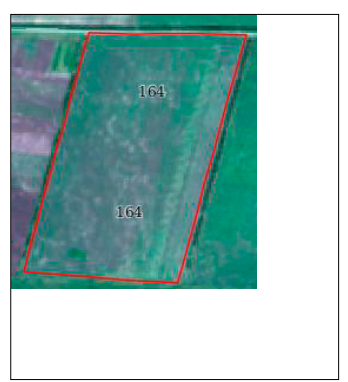

25.06.2018

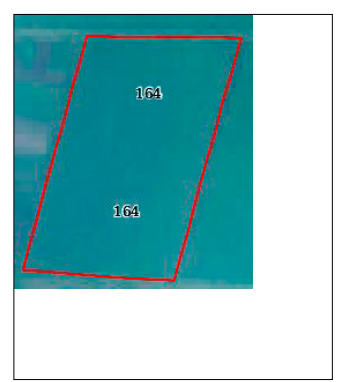

03.07.2018

Fig. 1. An example of an agricultural land object in the space image Sentinel-2A. Object No. 164 oats.

Figure 2 gives a fragment of a state change and transition diagram of the object under study in time. 


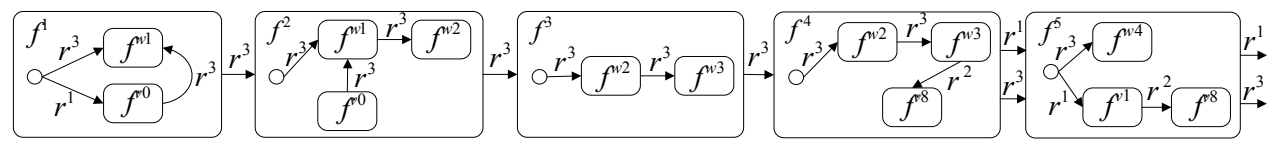

Fig. 2. A state change diagram of the object under study in time under the influence of natural and antropogenic factors.

The diagram depicts all possible states of the object corresponding to the reference time intervals. Thus, a composite state $f^{1}$, describing the object by the moment of sowing season beginning, includes the states $f^{v 0}$ and $f^{w 1}$, meaning that in the conditions of natural development of $r^{1}$ the object will turn to the state of overgrowing, and in case of AEM implementation $r^{3}$ it will turn to the state of harrowing, accordingly, which will be definitely reflected on the values of the spectral index.

Prior to the interpretation of a state of a field corresponding to $\Theta_{i}$ characterized by internal inhomogeneity, it is necessary to separate $\Theta_{i}$ into segments $w_{i}$ meeting the established criteria of homogeneity formed based on the values of NDVI:

$$
s(x, y)=\left\{\begin{array}{l}
\lambda_{1}=f^{w 1} \text { or } f^{w 6} \text { where } 0.025 \leq f(x, y) \leq 0.26, \\
\lambda_{2}=f^{w 2} \text { or } f^{w 3} \text { where } 0.26<f(x, y) \leq 0.43 \\
\ldots \\
\lambda_{6}=f^{v 4} \text { or } f^{v 5} \text { where } 0.72<f(x, y) \leq 0.85
\end{array}\right.
$$

where $f(x, y)$ is a brightness function of the original image, $s(x, y)$ is a segmented image, and $\lambda_{i}$ are segment marks, $i=0,1,2, \ldots, 6$.

The result of the segmentation is given in Figure 3 in the form of index images of the object under study.

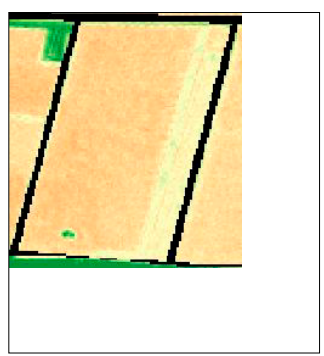

21.05 .2018

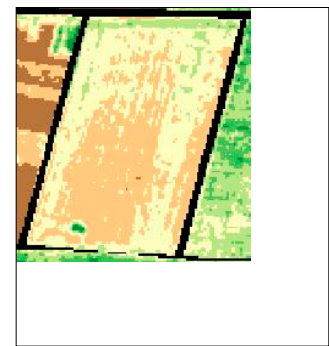

25.06.2018

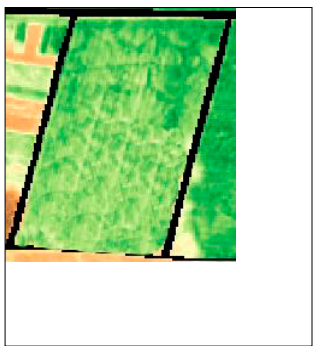

03.07.2018

Fig. 3. Index image of the object No. 164.

As distinct from $\Theta_{i}$ spatial coordinates of which are fixed, spatial position of $w_{i}$ shall be localized. For this purpose segmented areas $w_{i}$ are transformed from an iconic level to a level of geometrical and relational structures, where a feature vector formed for $w_{i}$ includes not only iconic features, but also metric characteristics physical interpretation of which enables us to describe a position, dimensions and form of inhomogeneities within the field (Fig. 4). This way $w_{i}$ can be considered and analyzed as an independent object. 


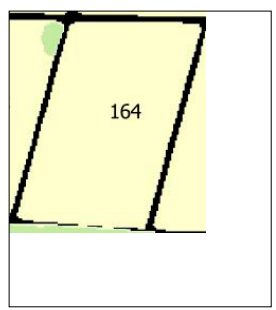

21.05.2018

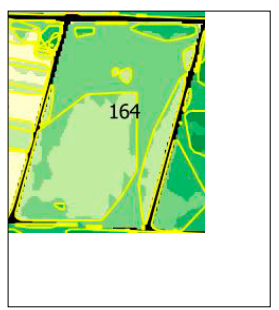

25.06.2018

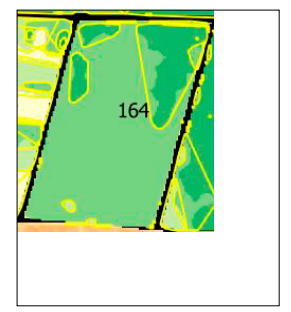

03.07.2018

Fig. 4. Segmented image of the object No. 164.

Metric characteristics are as follows:

1. Geographical coordinates in the given projection $P_{\Pi}=\left\{\left(x_{i}, y_{i}\right)\right\}$, describing the location of $w_{i}$.

2. Area of the object with polygonal geometric pattern:

$$
N S=\frac{1}{2}\left|\sum_{i=1}^{n-1} x_{i} y_{i+1}+x_{n} y_{1}-\sum_{i=1}^{n-1} x_{i+1} y_{i}-x_{1} y_{n}\right|
$$

where $\left(x_{i}, y_{i}\right), i=1,2, \ldots, n$ is a sequence of coordinates of the neighboring vertices of the polygon, $\left(x_{i+1}, y_{i+1}\right)=\left(x_{1}, y_{1}\right)$.

3. Perimeter of the object:

$$
N P=\sum_{i=1}^{n} \sqrt{\left(x_{i+1}-x_{i}\right)^{2}+\left(y_{i+1}-y_{i}\right)^{2}}
$$

where $i=1,2, \ldots, n$ is a number of vertices of the contour.

4. Thickness of the object:

$$
T=4 \pi\left(N S / N P^{2}\right)
$$

Thickness is a quantitative index characterizing the form of a non-heterogeneity contour. Under the ideal conditions, the form of the contour $w_{i}$ shall be convex, i.e. the value of $T$ shall be close to 1 . Yet, in practice heterogeneities possess borders with stretched broken form, i.e. $T$ tends to 0 .

In a result, an accompanying task emerges - to bring the inhomogeneity contour to the convex form, which in practice enables to organize parallel movement of agricultural machinery equipped with spotted treatment devices.

Bringing the contour to the convex form is based on Jarvis's Algorithm, which lies in the determination of a sequence of elements of a set of contour points forming a convex envelope for this set. The result of such conversion is given in Figure 4 during the analysis of object inhomogeneities for 25.06.2018 and 03.07.2018. The conversion covers only those non-homogeneous areas which, from the farmer's point of view, are appropriate for spotted treatment [3]. For this purpose, it is firstly necessary to take into account the minimum limitation of the non-heterogeneity area $-N S_{w i}^{*}$ which shall include the requirements for the width of suspended equipment used. Secondly, the accuracy of determining the belonging of a non-homogeneous area to this or that state is very important in the process of information support rendering.

To do that, it is firstly necessary to assess the deviation of index values of the nonhomogeneous area $w_{i}$ from the defined norm. Mean square deviation $\sigma$ is calculated for 
each inhomogeneity. After that, the probability of correct state determination is calculated. This calculation is based on the probability of mean index value $\bar{N}$ within the area $w_{i}$ falling into the diapason of values corresponding to the reference states of the object (4) typical of the current time $T m$ and is calculated using a formula of probability of a discrete random variable falling into the interval:

$$
P(\alpha<\bar{N} \leq \beta)=\Phi_{o}\left(\frac{\beta-d}{\sigma}\right)-\Phi_{o}\left(\frac{\alpha-d}{\sigma}\right),
$$

where $\alpha$ and $\beta$ are the defined threshold values of the intervals, $d$ is mathematical expectation, $\Phi_{o}$ is Laplace's function, $\bar{N}$ is calculated as follows:

$$
\bar{N}\left(w_{i}\right)=\frac{1}{n} \sum_{i=1}^{n} \operatorname{NDVI}\left(x_{i}, y_{i}\right),
$$

where $\operatorname{NDVI}\left(x_{i}, y_{i}\right)$ is a brightness function of the index image point.

In case $\sigma$ value for the non-homogeneous area $w_{i}$ is insignificant, the probability of this area falling into the diapason corresponding to a particular state is high and close to 1 . Therefore, the exactness of current actual state determination $f_{A j}^{i}\left(f_{A j}^{i} \in F\right)$ is close to $100 \%$. After that, current state of the area $w_{i}$ can be compared to a reference state. In case current state does not coincide with the reference one, whereby it is highly probable that the state of $w_{i}$ is determined correctly, it is recommended to examine the area $w_{i}$ under study.

In case $\sigma$ value for the non-homogeneous area $w_{i}$ is significant, the correctness of such area determination reduces greatly and an error increases.

In a result of the described analysis of a non-homogeneous dynamically changing structure of an agricultural land object throughout the whole vegetation period based on the space images, object-relational structures of an inhomogeneity presented in the form of inhomogeneity maps are formed (Fig. 4). Whereby, each non-homogeneous area $w_{i}$ of the $j$-th object is described using the following set of features enabling to compare a physical state of the $f^{i}$ field in the localized area $w_{i}$ in the image:

$$
w_{i}=<I D,\left\{P_{\Pi i}^{i}\right\}, N S_{j}^{i}, N P_{j}^{i}, T_{j}^{i}, \bar{N}_{j}^{i}, T m, k_{j}^{i}, e_{j}^{i}, \varphi_{j}^{i}, f_{A j}^{i}>,
$$

where $I D$ is a number or name of the agricultural land object.

\section{Conclusion}

A graph model of a spatial object "agricultural land" reflecting a variety of states and transitions caused by the abovementioned factors has been designed. The model accounts for the planned and actual development path of the object in time. It contains means of expression that describe the inhomogeneity of the object in time and space. The model has been used as the basis for agricultural monitoring system developed by the authors $[4,5]$. This system allows monitoring of the seasonal vegetation, localizing abnormal features in plants development, and monitoring of agro-engineering measures and their results to ensure information support of end users (agronomists, heads of agricultural enterprises and authorities). Based on this model, the system generates maps of inhomogeneities for each field at the set monitoring rate which allows for a comprehensive assessment of the object in time and timely revealing of adverse developments.

The reported study was funded by Russian Foundation for Basic Research (No.18-47242002), Government of Krasnoyarsk Territory, Krasnoyarsk Regional Fund of Science, to the research project: «The development of technology for creating intelligent information systems of object-oriented monitoring of territories based on remote sensing data». 


\section{References}

1. R. V. Brezhnev, Yu. A. Maglinets, K. V. Raevich, G. M. Tsybulsky, II International Conference: Information technology and nanotechnology, Section 2, 1090 (2018)

2. R. V. Brezhnev, Yu. A. Maglinets, CEUR Workshop Proceedings, 2033, 247 (2017)

3. R. V. Brezhnev, Yu. A. Maglinets, IV International Scientific Conference: Regional Problem of Earth Remote Sensing, 36 (2017)

4. R. V. Brezhnev, E. A. Mal'tsev, Pattern Recognition and Image Analysis, 25, 201 (2015)

5. R. V. Brezhnev, Yu. A. Maglinets, Journal of Sib. Fed. Univ. Engin. \& Techn., 10, 819 (2017) 